\title{
Generative Music Video Composition: Using automation to extend creative practice
}

\author{
Julia Stephan and Andrew R. Brown
}

Griffith University, Brisbane, Australia

\begin{abstract}
In this paper I outline the design of a system for dynamic real-time editing of music video sequences on the Internet, utilizing probabilistic parameters and algorithmic decision-making for progression. I will explain how this system enables music videos to be different every time they are accessed, thus providing users with an enhanced viewing experience and creators with a new tool for video composition. As the advancement of online technology influences the ever-changing habits of media consumers, user uptake of new technologies suggests that a transition away from video's prevalent mode of presentation, as a linear sequence of shots, is entirely possible. Factors such as viewer engagement and usability drive the need for ongoing exploration in the use of video for entertainment, information and advertisement. In this context, examples of innovations in music video production or presentation remain rare. The production of a music video with generative methods is described in this paper as a semi-automated process. Human tasks are not replaced by computational execution, but are shifted to become increasingly conceptual. A design for this novel mode of video composition is outlined and a creator's transformed experience when using a system that implements this design is described and compared to experiences with traditional methods of editing.
\end{abstract}

\section{Introduction}

Since the conception of the music video as a marketing tool for popular music in the 1980s, its visual aesthetic has been compared to the TV commercial and often described as being ephemeral, ambiguous and disjunctive (Frith et al 1993, Jhally 1995, Morse 1986, Vernallis 2004, Williams 2003). Goodwin (1992) argues that this serves the preservation of novelty in order to motivate repeated viewing, as the plot and meaning of a music video can rarely be fully grasped upon watching it only once. With recent developments in new media, it seems that the music video has undergone new modes of presentation to preserve novelty and stimulate viewers' curiosity. Examples like The Wilderness Downtown (2013), ROME (2013) and Lost in the Echo (2013) challenge the current technological limits and conventions of viewer engagement in online video. 
Stefan, J., \& Brown, A. R. (2014). Generative Music Video Composition: Using automation to ex-tend creative practice. Digital Creativity, Published online 15 June 2014. Retrieved from http://www.tandfonline.com/doi/abs/10.1080/14626268.2014.932289\#preview

Technology is providing the basis for the ever-changing nature of our media landscape and has sparked immense creativity in dynamic, adaptable and usercentered design, leading to the development of what Manovich (2001) calls "new media objects" - digital media elements that are moving away from linear presentation and pre-determined streams of information.

With the reduced importance of pre-determined music programming and new media trends in viral marketing that are orientated towards the individual's choice, it appears that any video must provide enough novelty and/or entertaining value to be shared online. This may be achieved by enhancing the viewers' experience, e.g., providing variation and involving them through interactive means. A degree of automation can help achieve these viewer outcomes as well being a means to extend an author's abilities.

As technology evolves to allow for broader and more stable access to the Internet and media rich applications, video is gradually becoming a more common element in web and application design and is moving beyond its status as a standalone feature.

One development in this context is the increasing integration of video content in social media. In early 2013 Vine, a new video sharing platform in the form of a mobile application was introduced. Vine allows users to record and share videos with a maximum duration of six seconds, which can be embedded on social networking platforms such as Twitter and Facebook (Sippey 2013). After acquiring the platform, Vine was promoted heavily by Twitter and its instant success was compared to that of the photo sharing application Instagram (Rohrer 2013, Taylor 2013). The videos' short duration and resulting small file size, among other aspects, make this kind of video content an ideal means for social interaction online. In July 2013 comparable video functionality was also added to Instagram (Systrom 2013). A similar concept of establishing a creative community around a short video format was used by Smule with the app CineBeat, released in December 2012 (Frost 2013). Cinebeat assembles recorded video footage into a 15-second music video, and transforms both sonic and visual qualities of the given video footage.

Another recent development regarding innovation in the field of online video at the time of this writing is YouTube's introduction of a feature that will make videos "shoppable", allowing viewers to click on hotspots in a video to directly access an online retailer's website where products seen in the video can be purchased (Tomassini 2013).

The implementation of video content as an essential media element of online services and sites can be risky due to potentially large file sizes and the resulting requirement for fast and stable Internet connections on the client-side. However, the potential of video content for marketing, social networking and information sharing is evident and it can be expected that this area of online media will become a 
Stefan, J., \& Brown, A. R. (2014). Generative Music Video Composition: Using automation to ex-tend creative practice. Digital Creativity, Published online 15 June 2014. Retrieved from http://www.tandfonline.com/doi/abs/10.1080/14626268.2014.932289\#preview

dominant subject of research and development. Cisco (2012) estimate that Internet video (excluding file sharing and gaming) will make up 55\% of all consumer Internet traffic by 2016, compared to general web/data traffic at $23 \%$ and file sharing at $21 \%$.

We assume that as a result of the expected rise in online video content, non-linear video applications will be employed within the next decade as a way to increase viewer engagement and usability.. We are therefore interested to developing a new way of creating and presenting online videos that corresponds to the changing needs posed by technology and media consumption.

More specifically, the aim of our research is the development of generative composition methods for music videos, to serve the purpose of creating a different video sequence every time it is accessed.

\section{Technology in the creative process}

The aforementioned technological advancements in new media allow us to introduce "metacreation" (Whitelaw 2004) into the video-editing process, which can potentially lead to new artistic forms for the moving image, as well as effective marketing instruments.

It is often suggested that new technologies can be regarded as tools that may enhance creativity and possibility by automating those tasks that are easily described by algorithms, thus leaving room for the creative mind to be given more complete expression. The concept of enhancing people's skills and abilities has been explored since the beginning of the digital age, particularly from the 1960s onwards, when fields such as cybernetics and Artificial Intelligence achieved widespread awareness. In 1960 Licklider (2003) described what he called the "man-computer symbiosis", in 1962 Engelbart (2003) spoke of "augmenting human intellect" and Nelson explained his vision called "Thinkertoys", as systems "to help people think", or more specifically as "a computer display system that helps you envision complex alternatives" (Nelson 1987 p. 50). In the 1970s Helvey (1971 p. 4) spoke of the "machine partner" and the necessity of "cybernetic pedagogy" in order to let human beings live adequately according to their times. He states that the study of cybernetics is essential to explore and provide the necessary means of communication between man and machine.

Using generative computing systems, an artist's activity may be transformed to decrease manual execution on one hand and increase conception on the other. McLuhan describes this process as creating "roles for people, which is to say depth of involvement in their work and human association that our preceding mechanical technology had destroyed" (2001 p. 7). McLuhan argues, following Heidegger (1977), that technology is inherent in human nature. Clark elaborates on this 
Stefan, J., \& Brown, A. R. (2014). Generative Music Video Composition: Using automation to ex-tend creative practice. Digital Creativity, Published online 15 June 2014. Retrieved from http://www.tandfonline.com/doi/abs/10.1080/14626268.2014.932289\#preview

subject, claiming that the unusual plasticity of the human brain leads to the extended use of tools and technology and a preference of "meta-knowledge over baseline knowledge" (Clark 2003 p. 67).

Our concept of generative music videos is highly dependent on the creation of appropriate software and a framework in which an editor can operate with this software. Following Dewey (1990), Brown (1999) suggests that computational systems and software as tools for artistic practice actively influence the creation of artefacts. In this context the primary motivations for the design of our system are to extend video production practices and enhance the outcomes of that practice. We expect that the system will influence the experience of both music video creation and consumption.

In the following sections we present our current framework for generative music video composition, whereby we highlight the author's new tasks and opportunities in the partly automated creation process.

\section{Generative music video editing}

We see video editing as an art form with a strong human-tool factor that defines the outcome of a film or video. Therefore, the revised relationship between human and machine in a renewed creative process that involves automated composition has to be examined closely. By introducing automated editing processes we intend to shift the emphasis of the human creative process toward the preparation of footage, shot pool selection, and management of the editing machine's decision making. Thus we arrive at several questions: How can editing conventions be efficiently described through algorithms? How is the process and experience of editing changed? By replacing a number of human-made decisions with machine-made ones, where might creative freedom get lost and where may it be gained?

A music video is assumed to be, put most simply, a sequence of various shots whose assembly depends on decisions of a technical, conventional and aesthetic nature. The shot is the basic building block, or granule, of the music video sequence. For use in a generative editing system, as outlined here, a filmmaker and/or editor produces a pool of shots that are to be assembled by algorithmic decision-making. Each shot has to provide the information, such as narrative and technical shot descriptions, needed to construct the plot. This information is part of a probabilistic structure of the music video's principal key characteristics that determines the progression of a sequence. The generation of sequences with this information and related probabilistic values is realized via greedy-type algorithms based on Markov chains.

An essential consideration to achieve coherence, meaning that the software is efficient on a technical level and accurate according to an author's intention for a music video's progression and rhythmic qualities, concerns the design of a 
Stefan, J., \& Brown, A. R. (2014). Generative Music Video Composition: Using automation to ex-tend creative practice. Digital Creativity, Published online 15 June 2014. Retrieved from http://www.tandfonline.com/doi/abs/10.1080/14626268.2014.932289\#preview

framework for shot description parameters and an algorithmic decision process that produces appropriate results.

\subsection{Parameters}

To support our method we identify two specific types of parameter that define the structure and progression of a generative music video; content-specific parameters and structural parameters.

Structural parameters comprise time definitions, such as maximum length of shots, segmentation and synchronization of events. These are fixed parameters and are essential to building the sequence on a basic level. They don't describe video content, but its temporal progression or "rhythm". Only their contained values may change and therefore they require minimal interaction from an editor. Contentspecific parameters concern narrative progression and aesthetic construction. These parameters are used for generating the narrative and influencing what may be perceived as a visual style in more detail by elaborating on the previously outlined basic structure. Content-specific parameters describe what can be seen in the shot, for example the camera distance, protagonists (musicians or fictional characters), or actions (performance of the song, narrative scenes, etc).

The precision of a parameter's value will reflect the potential variety of the given video characteristic. With camera distance, for example, we may want to describe a pattern with as much detail as possible.

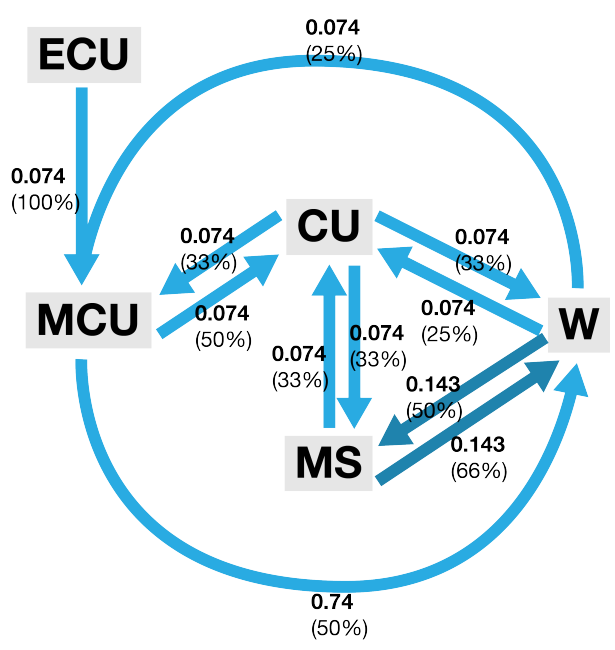

Figure 1: Probability model for camera distance

The implementation of five discrete parameter values, as seen in Figure 1, can, however, prove to be difficult, especially when combined with other parameters where such a complex distinction would not be seen as useful. For example,, from an 
Stefan, J., \& Brown, A. R. (2014). Generative Music Video Composition: Using automation to ex-tend creative practice. Digital Creativity, Published online 15 June 2014. Retrieved from http://www.tandfonline.com/doi/abs/10.1080/14626268.2014.932289\#preview

editor's perspective, it may be impossible or nonsensical to describe key characteristics with as many as five qualities. Furthermore, when using multiple parameters in the decision-process, the finding of a probable next shot can become impossible when the amount of footage doesn't accommodate this level of complexity. For example, given a decision process that works with three parameters and five qualities to each parameter, we arrive at 125 possible parameter combinations. Considering that our current experiments work with a range of 100200 shots per music video, it is unlikely that many of these combinations can be found at all in the shot pool, therefore there could end up being many empty spaces in too complex a transition matrix.

In order to support modularity, we want to standardize parameters and filtering processes by using the same structure for each parameter and only changing their values. A binary decision model was designed and tested (see Figure 2). In the example using camera distance, this would slightly alter the meaning of the way this parameter is implemented. Rather than creating a pattern of various camera distances, we define the occurrence of close-up shots as a key characteristic, and let the algorithm decide if the next shot is a close-up or not, rather than determining which particular camera distance is next.

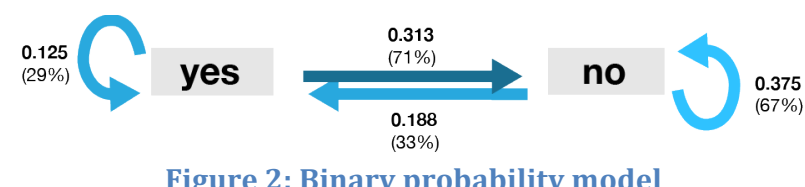

Figure 2: Binary probability model

With this model that reduces the parameter range, a larger number of parameters may be needed in order to make the algorithm's decision-making process more complex. In practice there exist many potential parameters, and so this is not an issue. By using this simplified binary base structure, video characteristics can be described as statements that are potentially more specific and intuitive to an author, both for initial creation and for testing a video's coherence.

\subsection{Decision process}

In order to predict the most appropriate following shot and subsequently to progress the sequence, an algorithmic process based on Markov chains is used to decide on a quality for each parameter according to pre-defined probabilities. In its simplest form the algorithm will loop through the provided footage to check each shot for these qualities and thereby create corresponding sets of candidate clips. Using the intersection of all sets, and random selection when required, one element is chosen to become the next shot. With a large amount of footage and /or a large number of parameters, this method can become problematic with regard to 
Stefan, J., \& Brown, A. R. (2014). Generative Music Video Composition: Using automation to ex-tend creative practice. Digital Creativity, Published online 15 June 2014. Retrieved from http://www.tandfonline.com/doi/abs/10.1080/14626268.2014.932289\#preview

processing speed which might, in real-time system, prevent uninterrupted playback. Additionally, measures must be taken to avoid generating disjoint sets.

The choice of parameters to implement must therefore be selective and defined in a hierarchical and/or categorized order. In our binary decision model we used a method of grouping content-specific parameters. One example is the use of the two groups "subject" and "context", whereby subject describes protagonists and active elements that drive the narrative, and context comprises parameters that make up surroundings and passive narrative elements. To minimize computing time, the shot pool is filtered using these parameter groups, rather than querying each parameter individually. Additionally, a simple hierarchy was implemented according to each group's potential autonomy in creating a coherent sequence. In case no intersections could be formed, the algorithm would fall back to the subject set and ignore context.

\subsubsection{Parameter relationships}

The introduction of parameter groups or categories allows for increased computing efficiency and coherence, but requires more conceptual input. An author must group parameters with their dependencies in mind.

Ideally, transition probabilities would enable optimal sequence decisions to ensure that choices are always safe. Given the algorithm's variability, this may not always be the case and therefore a feasibility function that is supported by defining and mapping out parameter dependencies is implemented, to secure coherence if conflicts or dead ends arise.

\section{Example}

Given independent probability in a group that contains three Boolean parameters, we arrive at $2^{3}$ or eight possible combinations, as shown in Table 1.

\begin{tabular}{|l|l|l|l|l|l|l|l|l|}
\hline Singer & 1 & 0 & 1 & 0 & 0 & 0 & 1 & 1 \\
Protagonist 1 & 1 & 0 & 0 & 1 & 0 & 1 & 0 & 1 \\
Protagonist 2 & 1 & 0 & 0 & 0 & 1 & 1 & 1 & 0 \\
\hline
\end{tabular}

We use an example, where the group "subject" comprises the parameters "singer", "protagonist 1" and "protagonist 2". We also assume a condition that limits the sample space of possible events from eight to six: protagonist 1 can only appear individually or with both, the singer and protagonist 2 , never with only one of the two. Figure 4 illustrates this condition. 
Stefan, J., \& Brown, A. R. (2014). Generative Music Video Composition: Using automation to ex-tend creative practice. Digital Creativity, Published online 15 June 2014. Retrieved from http://www.tandfonline.com/doi/abs/10.1080/14626268.2014.932289\#preview

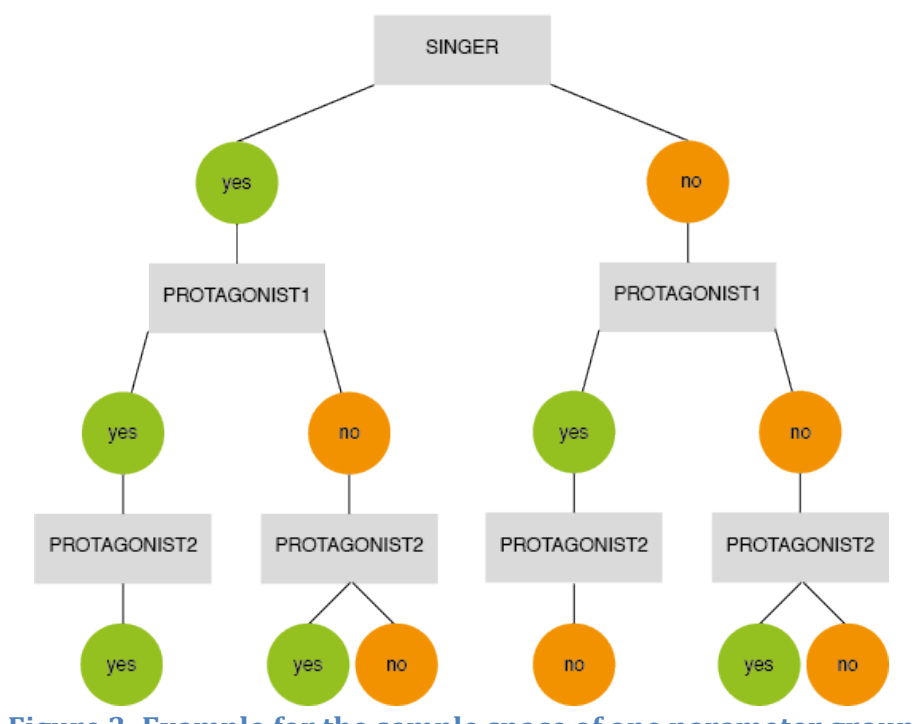

Figure 3: Example for the sample space of one parameter group

The second parameter group "context" describes three situations that can be shown in a shot: performance (a visual representation of the song being played), narrative activity (a supporting narrative is shown) and setting (the surroundings in which the video is taking place are shown individually, in this case, either showing protagonist 2 or no people at all). These parameters are mutually exclusive, which means that the sample space for this group consists of three events. The group is defined to be of secondary order, and therefore dependent on the subject group. Subsequently context events can only occur with particular combinations of events in the subject group, for example the occurrence of the singer implies that the context is either performance or a narrative element (see Figure 5). 
Stefan, J., \& Brown, A. R. (2014). Generative Music Video Composition: Using automation to ex-tend creative practice. Digital Creativity, Published online 15 June 2014. Retrieved from http://www.tandfonline.com/doi/abs/10.1080/14626268.2014.932289\#preview

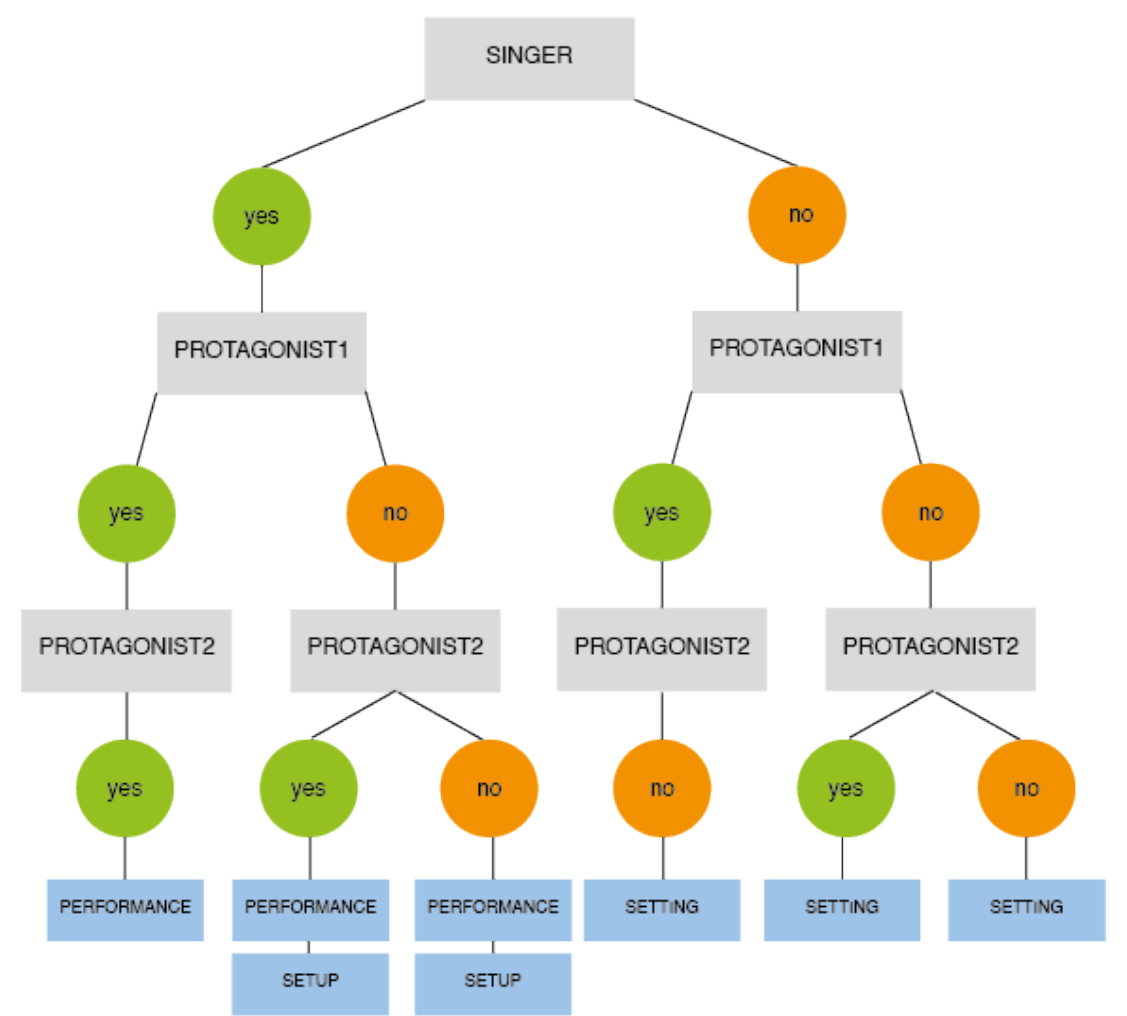

Figure 4: Example of the sample space of two parameter groups

\subsubsection{Segmentation}

Finally an author would have to make decisions about characteristic segments of the music video. This is based on the assumption that progressive and narrative dynamics of a video change throughout its duration according to musical structure or the narrative itself. To achieve this progressive change, probability values for clip selection can be assigned to particular sections of a song.

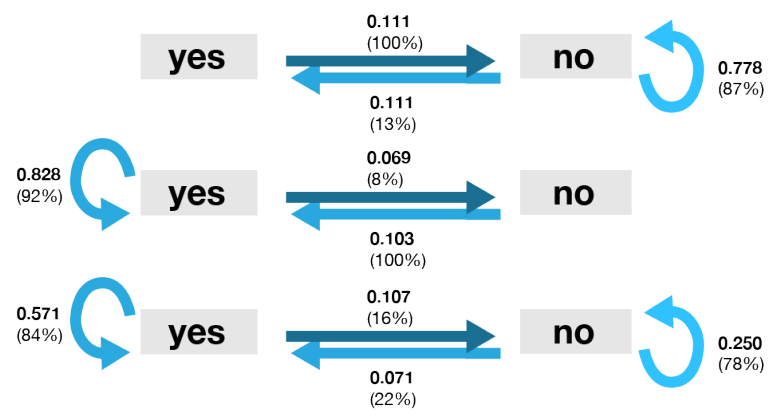

Figure 5: Segmented probability model

Figure 5 illustrates the dynamics of a given parameter divided into three segments. In comparison, using this parameter without segmentation would result in a much 
Stefan, J., \& Brown, A. R. (2014). Generative Music Video Composition: Using automation to ex-tend creative practice. Digital Creativity, Published online 15 June 2014. Retrieved from

http://www.tandfonline.com/doi/abs/10.1080/14626268.2014.932289\#preview

more generalized definition where most of the video's progression is left to chance rather than intention.

\subsection{Further considerations}

Our generative music video experiments are based on loose narratives that require limited definitions for temporal progression. The current framework therefore focuses on producing rhythm and a visualization of the musical experience to create a viewing mode that is "more like listening than viewing", in keeping with Vernallis' (2004 p.45) analysis of music video aesthetics. The implementation of more rigid narrative structures requires an additional parameter type that extends the model currently consisting of structural and content-specific parameters, as well as another step in the algorithmic decision-process.

Another aspect to consider for generative music video composition is the inclusion of rhythmic synchronization according to a song's beat. The current implementation can cover this aspect indirectly, depending on the number of defined segments and possible shot durations, which are able to create rhythmic patterns. Our editing analyses of recent music videos have shown that even in those videos that are perceived as highly orientated towards a song's structure and metric boundaries, cuts rarely appear exactly on the beat but are applied in combination with the visual content of a shot. Rather than implementing a full beat-orientated structure of cutpoints, we are using a method of identifying distinct structural moments of a song and assigning fixed clips, what we call "key-shots", to them. This means that certain shots are always played at the same time in a song, regardless of other parameters. With this method we are able to form a stronger connection between song and video and manage coherence without compromising too much of the generated sequence's variability.

In designing this system we also considered the possibility of integrating user interactivity and have designed the system to operate in real-time to allow for this possibility, even though at present interactivity is focused on control by an editor or designer rather than a viewer. In the course of this project we will limit interactivity to the influencing of salient parameter values, so an editor or viewer can decide to include more or less of selected characteristics. In this way an editor could, for example, decide to make a video more or less performance-driven or even turn off the use of performance shots altogether to only allow the viewing of shots related to the supporting narrative.

\section{Conclusion}

We have presented the basic framework of our generative music video system and outlined changes in the composition process, compared to the creation of linear video sequences. 
Stefan, J., \& Brown, A. R. (2014). Generative Music Video Composition: Using automation to ex-tend creative practice. Digital Creativity, Published online 15 June 2014. Retrieved from http://www.tandfonline.com/doi/abs/10.1080/14626268.2014.932289\#preview

With the intention of creating generating music videos that have the potential of being different every time they are accessed, we have employed a composition method based on Markov chains that assembles a sequence of video shots in realtime out of a pool of several hundred shots of varying durations.

The basis of our framework is the definition of different parameter types for the coding of clips and ensuring that these are vital to the design of algorithmic methods for decision-making, filtering and composition. Parameters consist of visual shot descriptions and their arrangement into probabilistic temporal structures. These features are the basis of algorithmic determination of shot selection and duration. To support computing efficiency and coherence of video sequences, the definition of individual parameters and subsequently the decision-process are implemented using binary logic. Due to the comparatively large number of parameters that may be needed for this method, we are using a hierarchical structure of parameter groups and parameter relationships within these groups to prioritise the probabilistic decision-making processes.

The system design has been focused on supporting an editor's processes of clip composition after footage has been filmed, edited and tagged with metadata. This approach underscores the highly conceptual activities an author must undertake to communicate his or her intentions for a given music video to the system before it can assemble the sequence. The editor must make logical connections between visual shot characteristics, plot and temporal progression on an increasingly abstract level.

It could be argued that a level of control is lost, by passing some responsibility for video composition to a machine, but we assert that with effective design of software and interfaces, video creators would find that the algorithmic editor extends their current abilities and offers new possibilities of adapting video content to viewer preferences. Although our proposed framework revolves to a large degree around automation, the influence of a human editor remains a crucial element in order to manage and influence algorithmic decision-making and judge the general quality of the generated sequences. As our research progresses, we intend to put a stronger focus on finding and defining an efficient and intuitive workflow that limits the requirement for technical knowledge regarding the computational background of our system. This workflow should support authors in finding and applying appropriate editing styles and narrative structures, while maintaining a leading creative position. By extension, it may also facilitate audience interaction with a system that dynamically produces unique music videos with each playback.

We suggest that our model of generative video composition can form one starting point of developing non-linear video applications, which could be of interest for innovation in various fields of new media. 
Stefan, J., \& Brown, A. R. (2014). Generative Music Video Composition: Using automation to ex-tend creative practice. Digital Creativity, Published online 15 June 2014. Retrieved from http://www.tandfonline.com/doi/abs/10.1080/14626268.2014.932289\#preview

\section{References}

All Is Not Lost, viewed 19 May 2013. <http://www.allisnotlo.st/>.

Brown A.R. 1999. Tools and Outcomes: Computer Music Systems and Musical

Directions. Imaginary Space: The proceedings of the Australasian Computer Music

Conference 16-22.

Cisco (2012). The Zettabyte Era. Viewed 19 May 2013.

<http://www.cisco.com/en/US/solutions/collateral/ns341/ns525/ns537/ns7

05/ns827/VNI_Hyperconnectivity_WP.html>.

Clark A. 2003. Natural-Born Cyborgs: Minds, Technologies, and the Future of Human

Intelligence. Oxford, New York: Oxford University Press.

Engelbart D. 2003. Augmenting Human Intellect, in N. Wardrip-Fruin \& Montfort N.

(ed.) The New Media Reader: 93-108.

Frith S., A. Goodwin \& L. Grossberg 1993. Sound and vision: The music video reader.

New York: Routledge.

Frost D. 2013. 'CineBeat' app by Smule makes music videos, San Francisco Chronicle, May 10, viewed 31 July 2013.

$<$ http://www.sfchronicle.com/technology/article/CineBeat-app-by-Smule-makes-

music-videos-4503906.php>.

Goodwin A. 1992. Dancing in the Distraction Factory: Music Television and Popular

Culture. Minneapolis, MN: University of Minnesota Press.

Heidegger M. 1977. The Question Concerning Technology and Other Essays. New York:

Harper \& Row.

Helvey T.C. 1971. The Age of Information: an Interdisciplinary Survey of Cybernetics.

Englewood Cliffs, N.J.: Educational Technology Publications.

Hickman L.A. 1990. John Dewey's pragmatic technology. Bloomington: Indiana

University Press.

Jhally S. 1995. Image-Based Culture: Advertising and Popular Culture, in G. Dines \&

Humez J.M. (ed.) Gender, race, and class in media: 77-87. Thousand Oaks, Ca.:

Sage Publications.

Licklider J.C.R. 2003. Man-Computer Symbiosis, in N. Wardrip-Fruin \& Montfort N.

(ed.) The New Media Reader: 73-82.

Lost In The Echo 2013. Viewed 19 May 2013. <http://lostintheecho.com/>.

Manovich L. 2001. The Language of New Media. Cambridge, Massachusetts: MIT Press.

McLuhan M. 2001. Understanding media: the extensions of man. London: Routledge.

Morse M. 1986. Postsynchronizing Rock Music and Television. Journal of

Communication Inquiry 10/15: 15-28.

Nelson T.H. 1987. Dream Machines, in T. Neldon, H. (ed.) Computer lib/Dream

machines: Redmond, Washington: Tempus Books of Microsoft Press.

Rohrer F. 2013. Vine: Six things people have learned about six-second video in a week,

BBC News Magazine, 31 January, viewed 9 July 2013.

$<$ http://www.bbc.co.uk/news/magazine-21267741>.

ROME. Viewed 19 May 2013, <http://www.ro.me/>. 
Stefan, J., \& Brown, A. R. (2014). Generative Music Video Composition: Using automation to ex-tend creative practice. Digital Creativity, Published online 15 June 2014. Retrieved from http://www.tandfonline.com/doi/abs/10.1080/14626268.2014.932289\#preview

SIPPEY M. 2013. Vine: A new way to share video, Twitter Blog, 24 January, viewed 9 July 2013, < https://blog.twitter.com/2013/vine-new-way-share-video>.

Sүзтвом K. 2013. Introducing Video on Instagram, Instagram blog, 20 June, viewed 9 July $2013,<$ http://blog.instagram.com/post/53448889009/video-on-instagram>.

TAYLOR C. 2013. What Makes Vine So Hot? Mashable, 30 January, viewed 9 July 2013, $<$ http://mashable.com/2013/01/29/vine/>.

The WiLDERness Downtown 2013, viewed 19 May 2013, $<\mathrm{http}: / /$ thewildernessdowntown.com/>.

Tomassin D. 2013. Connecting people and the products they love, Google Retail Advertising Blog, 15 May, viewed 19 May 2013, $<$ http://googleretail.blogspot.com.au/2013/05/connecting-people-and-products-theylove.html $>$.

Vernaldis C. 2004. Experiencing Music Video: Aesthetics and Cultural Context. New York: Columbia University Press.

Whitelaw M. 2004. Metacreation: Art and Artificial Life. Cambridge, Massachusetts: MIT Press.

WiLliams K. 2003. Why I [Still] Want My MTV. Cresskill, NJ: Hampton Press, Inc. 